\title{
Timing and volume of information relevant to the Otolaryngologist during the COVID-19 pandemic in the UK.
}

\author{
Cristina Cernei ${ }^{1}$, Manu Shrivastava ${ }^{1}$, William Colquhoun-Flannery ${ }^{2}$, and stuart winter ${ }^{1}$ \\ ${ }^{1}$ John Radcliffe Hospital \\ ${ }^{2}$ Royal Berkshire NHS Foundation Trust
}

July 16, 2020

\begin{abstract}
Background: The SARS-CoV-2 was first reported in December 2019 in Wuhan, China and has been declared a pandemic in March 2020. COVID-19 has caused unprecedented and lasting biopsychosocial effects worldwide. All healthcare professionals have faced life threatening risks by attending their daily jobs. The daily emergence of advice and guidelines was necessary to ensure the safety of patients and staff. To this effect, all elective services came to a halt to preserve hospitals' capacity for dealing with the sickest. This retrospective, descriptive review aims to assess the volume and timing of the advice released specifically relevant to the UK Otolaryngologist. Methods: The search included online advice published in English by international, national and ENT-specific organisations between the 1st of January to the 31st of May. The date, title, source, type of advice and link to the advice was recorded in Excel. The resources were analysed per week of publication. A separate search for peer-reviewed publications was conducted using PubMed Central and Cochrane databases. Findings: 175 covid-19 related guidance's were identified. $52 / 175(29.7 \%)$ articles were published by international organisations. 56/175 (32\%) were produced by national organisations and $67 / 175(38.28 \%)$ were produced by ENT specific organisations. The peak guidance production took place in the third and fourth week of March (16/03/2020- 29/03/2020) with 72/175 publications. Of these, 27/72 came from the international category, 17/72 from national bodies and 28/72 from ENT-specific organisations. 13,863 total publications relating to COVID-19 were found using PubMed and Cochrane search strategies; $76 \%$ were relevant to ENT surgeons. Conclusion: The challenges faced by the Otolaryngologist relate to the unprecedented, sudden and daily changes to clinical practice. Multiple bodies interpreted the guidance giving an opportunity for confusion and delays in treatments for patients. Implementing a system with clear lines of communication and dissemination of information will improve our response in future pandemic events whilst maintaining a commercial awareness to better use the human and financial resources of an already financially-restricted NHS.
\end{abstract}

\section{Introduction}

The COVID-19 pandemic caused by the SARS-CoV-2 coronavirus infection has challenged the world's healthcare systems in an unprecedented way ${ }^{1,2}$. In the UK and wider world there has been a lot of discussion about how to change the shape of the SARS-CoV-2 incidence and associated mortality curve. The aim of this study is to look at the shape and timing of the information curve, in particular with reference to Otolaryngology in the UK. Although the broad principles may apply to other specialities and in other countries.

The result of the pandemic has led to a significant reduction in GP cancer referrals, elective Otolaryngology and cancer surgery ${ }^{3-5}$. This reduction is due to a number of factors, including access to health care, repurposing theatre suites, ventilators and staff in addition to evidence that suggesting an unacceptably high morbidity and mortality if patients contract COVID 19 in the perioperative period ${ }^{6-9}$.

In addition, there has been significant concern as to the risks to the surgical team ${ }^{10}$. Early evidence emerged that surgeons, in particular otolaryngologists, as well as other professionals operating on the aerodigestive 
tract were at particularly high risk. This is due to the high level of aerosol generated procedures involved in the speciality. A number of fatalities among Otolaryngologists and other similar practitioners has been reported $^{4,11}$.

During the pandemic there has been a significant number of guidelines produced by international sources, UK governmental organisations as well as specialist bodies such as the Royal Colleges and specialist organisations $4-6,12-17$.

These guidelines have dealt with a number of aspects including personal protective equipment, prioritisation of treatment and recovery. As well as specific aspects of Otolaryngology practice, including cancer, clinics, tracheostomy, rhinology, otology and paediatrics ${ }^{4-6,12-17}$.

The volume and quantity of guidelines has resulted in significant challenges for the Otolaryngologist to stay up to date and to be able to incorporate the advice into safe practice both for the patient and the clinician. In addition to the published advice the Otolaryngologist has been required to assimilate guidance produced by local hospitals, as well as considering governmental briefings, web-based discussion, the press and social media.

Not all of this advice has been consistent and high-level advice from international and national sources has been difficult to apply to specific procedures and settings.

This study aims to document the volume and timing of advice pertinent to the Otolaryngologist. This may help to inform new systems in the future if there are 'second' waves or future pandemics.

\section{Materials and Methods}

The search covered online advice published in English by official sources between the 1st of January and $31^{\text {st }}$ of May. The sources were broadly grouped in international, national and ENT-specific organisations.

The international resources included the World Health Organisation (WHO) and other relevant groups publishing advice relevant to ENT and surgical services (Centre for Medicare and Medicaid Services, American Academy of Otolaryngology and Head and neck, Stanford Group,ENT Canada, American College of Surgeons). The Covid-19 advice page compiled by the Cochrane group was used to select relevant international resources (https://ent.cochrane.org/news/covid-19-coronavirus-disease-ent-hearing-balance) .

National resources included advice from Public Health England (PHE), National Institute for Clinical Excellence (NICE), National Health System website (NHS), Academy of Medical Royal Colleges, Royal colleges (of Surgeons, Radiologists, Pathology, Speech and Language therapy).

ENT-specific organisations included ENT-UK, British Association of Head and Neck Oncologists (BAHNO), British Laryngology Association (BLA), British Rhinological Society, British Otological Society.

Excel was used to record the data by date, title, publishing organisation, type of advice and link. The type of advice referred to areas of concern during the pandemic - PPE, Service prioritisation, General (advice on treatment and/or investigations), cancer, tracheostomy, otology, rhinology, paediatric ENT and service recovery.

The volume of advice published weekly in each of the three main categories - international, national and ENTspecific organisations was reviewed. The number of SARS-CoV2 related deaths recorded during the above period were extracted from the governmental website (reference). This was used to analyse the trajectory of Covid-19 related deaths and the volume of advice published between January and June 2020.

The electronic databases PubMed Central and Cochrane were searched comprehensively from the 30th December 2019 to 31st May 2020. The first search identified all publications related to COVID-19, filtering duplicates and then calculating the number of publications by week.

Next, a subset of these COVID-19 papers relating to ENT was identified. These papers were categorised according to those describing subspecialties within ENT, common ENT symptoms, anatomical areas relevant 
to ENT, and research relating to personal protective equipment, service prioritisation and recovery following the COVID-19 pandemic.

The official UK government website was used to map daily laboratory-confirmed cases in England and UK deaths across the same time period ((https://coronavirus.data.gov.uk/?_$g a=2.237121091 .2105726748 .1588817632$-725862875.1586435801).

Ethical approval was not required because this study was a review and all data

were extracted from published articles

\section{Results}

A total of 194 Covid-19 related guidance relevant to the Otolaryngologist was identified during the 20 weeks between the 1st of January and $31^{\text {st }}$ May 2020. 19 of these 194 duplicates published by multiple organisations as collaborative work. Therefore 175 original articles were published by international organisations 52/175 (29.70\%), national organisations 57/175 (32\%) and by ENT specific organisations 66/175 (38.20\%). Figure 1.

All of the 175 guidance's covered multiple topics, separating these into the 10 main topics PPE was the subject in $31 \%$, Service prioritisation (13\%). General advice relating to treating elective otolaryngological conditions either surgically or conservatively (20\%). Screening (4\%), Cancer care (8\%), tracheostomy (7\%) and specific areas of Otology (4.1\%), Rhinology (4\%), Paediatrics ENT (3\%) and Service recovery (5\%). In total 313 topic areas were covered in the 175 guidance's. (Table 1)

The WHO declared the pandemic on the 11th March $2020^{18}$. Before this date only 8/175 guidance had been published. Following this there was a rapid escalation in guidance being issued. The peak guidance production took place in the third and fourth week of March (16/03/2020- 29/03/2020) with 33/175 publications in the third week of March and 37/175 in the fourth week. (See Figure 2)

Of these 70 publications, 27 came from the international category, 17 from national bodies and 26 from ENT-specific organisations. During this peak period the majority of the advice was related to PPE (48/70, $68.5 \%$ ). (Table 2)

Service recovery related publications commenced a fortnight post-peak guidance period. Between the $9^{\text {th }}$ of April and the $20^{\text {th }}$ of May, 16 guidances on service recovery were identified. During this period, the number of publications increased by an average of 2.2 per week with a peak of 5 publications during the second week of May.

12 came from national and ENT-specific bodies: 5 from ENT-UK, 2 from the Royal College of Surgeons, 3 from Public Health England, 1 from the Royal College of Radiologists and 1 from the NHS website. 4 came from International bodies included the American Academy of Otolaryngology and Head and Neck, Centres for Medicare and Medicaid.

Most guidance relevant to otology, rhinology and paediatric ENT was produced during the peak period and constitutes $10 \%, 7.1 \%$ and $7.1 \%$ respectively. During the subsequent four weeks $1-2$ pieces of advice were produced per week before the number of publications tailed off.

A total of 13,863 peer-reviewed publications relating to COVID-19 were identified using PubMed and Cochrane searches. At the time of the pandemic being announced there had been 966 COVID-19 related publications of which 610 related to Otolaryngology. The volume increased over time, with a sharp increase occurring at the end of March 2020. Figure 3. A similar pattern was followed by the volume of publications relevant to ENT, where 10,567 total publications were identified.

\section{Discussion}

The WHO declared the COVID-19 infection as a global pandemic on the 11th March $2020^{18}$. This study documents the advice that the UK-based Otolaryngologist would need to read and assimilate to prioritise, 
protect and triage patients as well as staff during this period.

As we emerge from the first wave of this unprecedented world-wide pandemic, we must learn lessons. Since the start of this century, there have been a number of epidemics; all have arisen outside the UK, including Ebola, severe acute respiratory syndrome (SARS), H1N1 influenza pandemic, Middle East Respiratory Syndrome, or MERS, and H5N1

https://www.who.int/csr/disease/ebola/ebola-6-months/myths/en/.

The COVID-19 pandemic represents an opportunity to analyse retrospectively the UK's response in terms of the volume and timing in which information was produced.

The virus initially described in Wuhan has spread throughout the world and, while the infection was recognised as a pandemic on the 11th March, however UK incidence numbers did not start to rise significantly until later in March with a probable peak in mid-April 2020. By the time the infection was recognised as a global risk, there had been a significant number of cases reported worldwide.

Otolaryngologists and other medical practitioners performing aerosol-generating procedures (AGPs) were identified early on as being at a high risk ${ }^{4,10}$. While this study is specific to this subset of medical practitioners, the volume of advice and the time it was produced is likely to be applicable to other specialties.

This study has identified 175 guidance publications issued from a number of international, governmental and specialist groups relevant to the Otolaryngologist. It is likely that this is an underestimate of the total guidance produced. Fifty-two documents came from international groups including the WHO and international ENT groups. Fifty-seven were produced by national organisations, including NHS Eng, Public Health England (PHE), royal colleges (RCS England, RCS Edinburgh, RCPath, RCRadiology, RCP) and various surgical societies and associations. Specialist bodies including ENT-UK, BAHNO, BOS, BRS also produced 66 pieces of advice.

The subject of that advice covered a number of topics including PPE, service provision, general provision of care, cancer-specific management, screening and recovery as well as specific aspects of Otolaryngology, including tracheostomy, otology, rhinology and paediatrics.

During this period medical services have been required to rapidly redevelop to allow for appropriate provision of care for COVID-19 infected patients. There has also been a need to limit hospital visits, rationalise procedures and limit surgical operations ${ }^{16,19}$. The latter reflects the risks to patients contracting COVID19 in the perioperative period and the high rate of pulmonary complications and mortality. In addition to protecting patients, there has been considerable guidance on how best to protect medical staff. As a consequence, a large number of clinical pathways have been changed. The long-term impact of such changes remains to be assessed ${ }^{7,20}$.

The peak time for guidance production in the UK was between the $16-29^{\text {th }}$ March 2020. During this time numbers of cases of COVID-19 in the UK were rising rapidly. Specific advice regarding clinical aspects of care required an assimilation of publicly available information, published data and international experience. This review illustrates that there was a number of rapidly published guidelines which informed clinical practice. Limitations of timescale mean that guidelines have not always been evidence-based and have been formulated by opinion and consensus, often relying on collaborative approaches ${ }^{21-24}$.

Peer review publications have followed the pandemic, with an estimated 13, 863 COVID-19-related publications of which $76 \%$ were relevant to the Otolaryngologist. It can be seen that there has been an unprecedented number of publications published during the pandemic. Also during this time many publications have been published using preprint servers and promoted on social media before peer review and even after peer review at least two high profile journals have had to retract publications ${ }^{25}$. This reflects the significance of the event but also challenges in medicine to understand and utilise this information.

With so much evidence produced in a short time, while infection rates were climbing was a huge challenge to incorporate into safe clinical practice. Moreover, translating high-level international guidance into meaningful 
advice for a given procedure in a particular setting is difficult. It is therefore unsurprising that there was some confusion.

This study is illustrative of the challenges faced by the Otolaryngologist in absorbing, assimilating, and implementing the advice produced. Much of the advice was produced while the numbers of new cases were increasing. While this is specific to the Otolaryngologist, it is likely that this pattern of advice and timing is relevant to other specialities operating in different regions of the world.

Within the UK much of the discussion has been about flattening the curve of infections. This study suggests that there is also a need to move the information curve to the left. Having a system that identified the impending pandemic would allow for the preparation of clear guidelines before the number of cases started to increase.

There is also a need to flatten the information curve. National and international guidance advice needs to be assimilated into advice that relates to specific procedures considering the physical setting they will occur in, local practice and availability of equipment. Having multiple bodies interpreting the guidance is an opportunity for confusion. Ultimately, this may result in a failure to follow the guidance, potentially putting medical personnel or patients at risk, or an over-interpretation of advice resulting in treatment being withheld.

If a pattern of infections arose again like the COVID-19 pandemic then there are opportunities if mechanisms are in place to generate and co-ordinate the advice needed by Otolaryngologists as well as other medical teams so that the advice can be available before numbers of infections start to rise. Much like the early warning system introduced after the 2004 Tsunami, there is a need to establish a similar system in the UK with clear lines of communication and dissemination of information.

\section{References:}

1. Fontanarosa PB, Bauchner H. COVID-19-Looking Beyond Tomorrow for Health Care and Society. JAMA 2020; 323 (19): 1907-8.

2. Zhu N, Zhang D, Wang W, et al. A Novel Coronavirus from Patients with Pneumonia in China, 2019. $N$ Engl J Med 2020; 382 (8): 727-33.

3. Mahase E. Covid-19: Urgent cancer referrals fall by $60 \%$, showing "brutal" impact of pandemic. $B M J$ 2020; 369 : m2386.

4. Givi B, Schiff BA, Chinn SB, et al. Safety Recommendations for Evaluation and Surgery of the Head and Neck During the COVID-19 Pandemic. JAMA Otolaryngol Head Neck Surg 2020.

5. Collaborative C. Global guidance for surgical care during the COVID-19 pandemic. Br J Surg 2020.

6. Couloigner V, Schmerber S, Nicollas R, et al. COVID-19 and ENT Surgery. Eur Ann Otorhinolaryngol Head Neck Dis 2020;137 (3): 161-6.

7. Day AT, Sher DJ, Lee RC, et al. Head and neck oncology during the COVID-19 pandemic: Reconsidering traditional treatment paradigms in light of new surgical and other multilevel risks. Oral Oncol2020; 105: 104684 .

8. Lei S, Jiang F, Su W, et al. Clinical characteristics and outcomes of patients undergoing surgeries during the incubation period of COVID-19 infection. EClinicalMedicine 2020: 100331.

9. Nepogodiev D, Glasbey JC, Li E, et al. Mortality and pulmonary complications in patients undergoing surgery with perioperative SARS-CoV-2 infection: an international cohort study. The Lancet 2020.

10. Bandyopadhyay S, Baticulon R, Kadhum M, et al. Infection and mortality of healthcare workers worldwide from COVID-19: a scoping review. medRxiv; 2020. 
11. Vukkadala N, Qian ZJ, Holsinger FC, Patel ZM, Rosenthal E. COVID-19 and the Otolaryngologist: Preliminary Evidence-Based Review.Laryngoscope 2020.

12. Mehanna H, Hardman JC, Shenson JA, et al. Recommendations for head and neck surgical oncology practice in a setting of acute severe resource constraint during the COVID-19 pandemic: an international consensus. The Lancet Oncology 2020.

13. Argenziano M, Fischkoff K, Smith CR. Surgery Scheduling in a Crisis. New England Journal of Medicine 2020; 382 (23): e87.

14. Head MA, Consortium NSTG, members C, et al. Head and neck surgical oncology in the time of a pandemic: Subsite-specific triage guidelines during the COVID-19 pandemic. Head $\mathscr{E}$ Neck 2020; 42 (6): 1194-201.

15. Paleri V, Hardman J, Tikka T, Bradley P, Pracy P, Kerawala C. Rapid implementation of an evidencebased remote triaging system for assessment of suspected referrals and patients with head and neck cancer on follow-up after treatment during the COVID-19 pandemic: Model for international collaboration. Head Neck 2020.

16. Dharmarajan H, Anderson JL, Kim S, et al. Transition to a virtual multidisciplinary tumor board during the COVID-19 pandemic: University of Pittsburgh experience. Head $\mathscr{E}$ Neck 2020; 42 (6): 1310-6.

17. Shuman AG, Campbell BH, Ethics A, Service P. Ethical framework for head and neck cancer care impacted by COVID-19. Head $\mathscr{E}$ Neck2020; n/a (n/a): 1214-7.

18. General WHOD. WHO Director-General's opening remarks at the media

briefing on COVID-19 - 11 March 20202020.

19. Workman AD, Welling DB, Carter BS, et al. Endonasal instrumentation and aerosolization risk in the era of COVID-19: simulation, literature review, and proposed mitigation strategies. Int Forum Allergy Rhinol 2020.

20. Richards M, Anderson M, Carter P, Ebert BL, Mossialos E. The impact of the COVID-19 pandemic on cancer care. Nature Cancer 2020;1 (6): 565-7.

21. Thomson DJ, Palma D, Guckenberger M, et al. Practice Recommendations for Risk-Adapted Head and Neck Cancer Radiation Therapy During the COVID-19 Pandemic: An ASTRO-ESTRO Consensus Statement. Int J Radiat Oncol Biol Phys 2020.

22. Topf MC, Shenson JA, Holsinger FC, et al. Framework for prioritizing head and neck surgery during the COVID-19 pandemic. Head \& Neck2020; n/a (n/a): 1159-67.

23. Yan F, Nguyen SA. Head and neck cancer: high-risk population for COVID-19. Head $\mathscr{E}$ Neck 2020; 42 (6): 1150-2.

24. Kowalski LP, Sanabria A, Ridge JA, et al. COVID-19 pandemic: Effects and evidence-based recommendations for otolaryngology and head and neck surgery practice. Head Neck 2020; 42 (6): 1259-67.

25. Wise J. Data transparency: "Nothing has changed since Tamiflu".BMJ 2020; 369 : m2279.

Tables:

\begin{tabular}{ll}
\hline Area & Advice area covered total 313 \\
PPE & 97 \\
Service prioritisation & 41 \\
General (treatment, investigations and/or prevention) & 63 \\
Screening & 13 \\
Cancer & 26
\end{tabular}


Tracheostomy 22

Otology 13

Rhinology 13

Paediatric ENT $\quad 9$

Service recovery $\quad 16$

Table 1: Subject of the advice covered by the Covid-19 related guidance relevant to the Otolaryngologist between the 1st of January and $31^{\text {st }}$ May 2020.

\begin{tabular}{ll}
\hline Area & Number of publications, $\mathrm{N}=70$ \\
$\mathrm{PPE}$ & 48 \\
Service prioritisation & 16 \\
General (treatment, investigations and/or & 29 \\
prevention) & 7 \\
Screening & 13 \\
Cancer & 13 \\
Tracheostomy & 7 \\
Otology & 5 \\
Rhinology & 5 \\
Paediatric ENT & 0 \\
Service recovery & \\
\hline
\end{tabular}

Table 2. Advice subject covered by the 70 Covid-19 related guidance relevant to the Otolaryngologist during the peak period $(16 / 03 / 2020-29 / 03 / 2020)$

Supplementary table 3: COVID-19 search strategy, using the PubMed database.

\begin{tabular}{ll}
\hline No. & Search \\
$\# 1$ & covid-19' OR 2019-ncov OR "sars-cov-2" \\
$\# 2$ & "wuhan coronavirus" OR "novel coronavirus" \\
$\# 3$ & 1 or 2
\end{tabular}

Supplementary table 4: ENT-specific search strategy (of publications identified by COVID-19 search)

\begin{tabular}{lll}
\hline No. & Theme & Search \\
$\# 1$ & Specialty & Otolaryngolog* OR ENT OR otorhinolaryngology OR rhinolog* OR otolog* OR audiolog* \\
$\# 2$ & Symptom & pharyngodynia OR nasal OR rhinorrhoea OR smell OR anosmia OR hyposmia OR dysgeusia OR ta \\
$\# 3$ & Anatomy & head and neck OR tracheotomy OR tracheostomy OR trachea OR tracheal OR larynx OR laryngeal \\
$\# 4$ & PPE & mask OR masks OR facemask OR facemasks OR N95 OR N-95 OR N97 OR N99 OR FFP OR respi \\
$\# 5$ & Prioritisation & surgery OR surgical OR surgeries OR surgeon OR surgeons OR operate OR operation OR operation \\
$\# 6$ & Recovery & discharge OR discharges OR discharging OR discharged OR stepdown OR re-open OR re-opening O \\
$\# 7$ & & 1 OR 2 OR 3 OR 4 OR 5 OR 6 \\
\hline
\end{tabular}

Figures: 


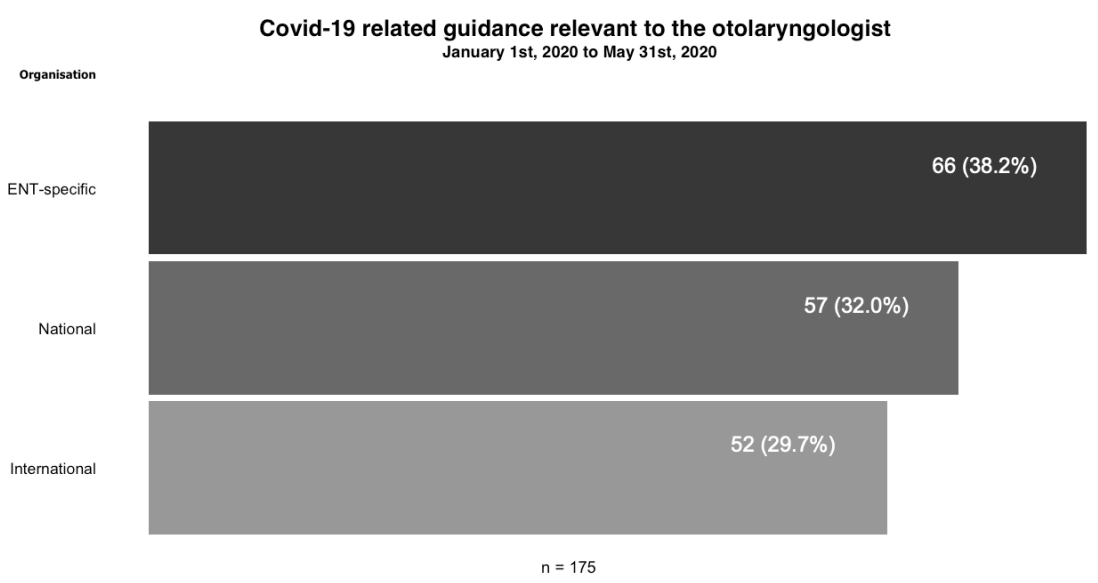

Figure 1. Covid-19 related guidance relevant to the otolaryngologist (January $1^{\text {st }}, 2020$ to May $31^{\text {st }}, 2020$ )

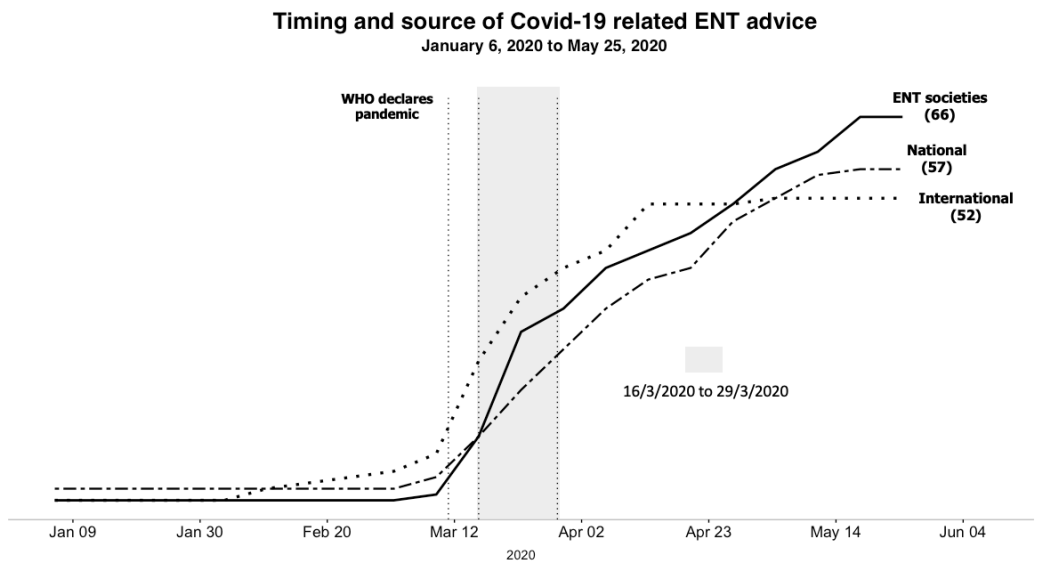

Figure 2. Timing and source of UK advice relating to Covid-19 by organisation between the $6^{\text {th }}$ January and 25th May 2020 


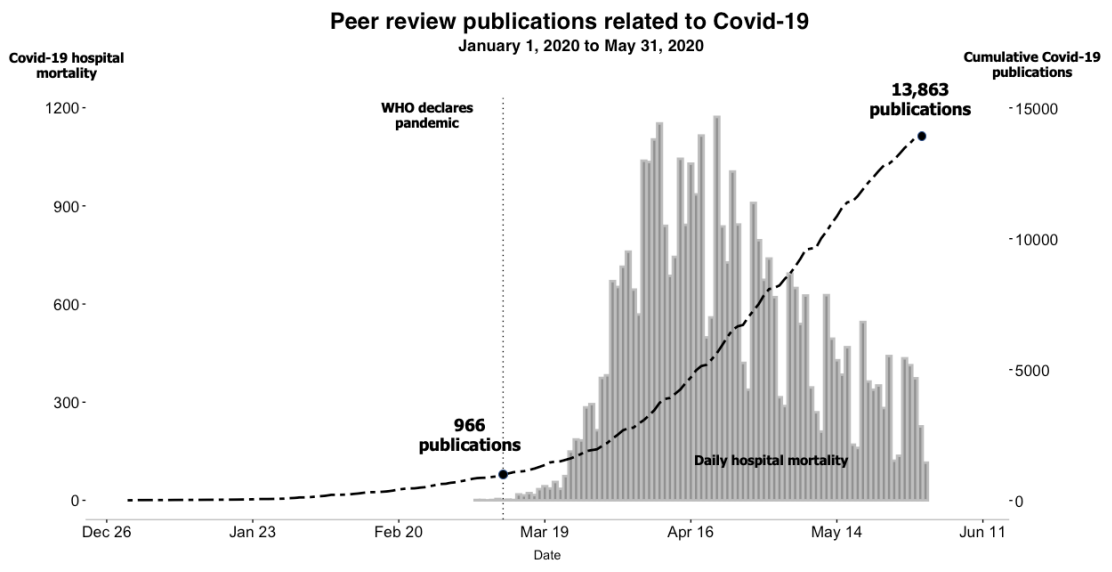

Figure 3. Peer review publications related to Covid-19 (January 1, 2020 to May 31, 2020) 